\title{
Stage IIA Ampulla of Vater Cancer AJCC v8
}

National Cancer Institute

\section{Source}

National Cancer Institute. Stage IIA Ampulla of Vater Cancer A/CC v8. NCI Thesaurus.

Code $C 134870$.

Stage IIA includes: T3a, N0, M0. T3a: T umor directly invading the pancreas (up to 0.5

cm). NO: No regional lymph node metastasis. M0: No distant metastasis. (AJCC 8th ed.) 